Pacific Journal of Mathematic 


\title{
ASYMPTOTIC PROPERTIES OF NONOSCILLATORY SOLUTIONS OF HIGHER ORDER DIFFERENTIAL EQUATIONS
}

\author{
W. J. KIM
}

\begin{abstract}
A classification of the nonoscillatory solutions based on their asymptotic properties of the differential equation $y^{(n)}+p y=0$ is discussed. In particular, the number of solutions belonging to the Kiguradze class $A_{\jmath}$ is determined.
\end{abstract}

We investigate asymptotic properties of the nonoscillatory solutions of the differential equation

$$
y^{(n)}+p y=0,
$$

where $p$ is a continuous function of one sign on an interval $[a, \infty)$. Various aspects of Eq. (E) have been investigated by a number of authors [1-15]; in most cases, under the condition that the integral

$$
I(r) \equiv \int_{a}^{\infty} x^{r}|p(x)| d x
$$

is either finite or infinite for some constant $r$. For instance, Eq. (E) is oscillatory on $[a, \infty)$ if the integral (1) is infinite with $r=n-1-\varepsilon$ for some $\varepsilon>0[4,8]$. On the other hand, if $I(n-1)$ is finite, (E) is nonoscillatory; in fact, it is eventually disconjugate [9, 14, 15]. Under the same condition, results on the existence of a fundamental system of solutions possessing certain asymptotic properties have also been obtained [5, 13]. Of particular interest to the present work, however, is the notion of class $A_{p}$ introduced by Kiguradze [4] with the help of inequalities in Lemma 1.

A solution of $(\mathrm{E})$ is said to be nonoscillatory on $[a, \infty)$ if it does not have an infinite number of zeros on $[a, \infty)$. (Unless the contrary is stated, the word "solution" is used as an abbreviation for "nontrivial solution.") Eq. (E) is said to be nonoscillatory on $[a, \infty)$ if every solution of $(\mathrm{E})$ is nonoscillatory on $[a, \infty)$. If there exists a point $b \geqq a$ such that no solution of (E) has more than $n-1$ zeros on $[b, \infty)$, Eq. (E) is said to be eventually disconjugate on $[a, \infty)$.

As previous studies of Eq. (E) indicate, asymptotic properties of the solutions strongly depend on the parity of $n$ and the sign of $p$. For this reason, it is convenient to classify Eq. (E) into the following four distinct classes:

$$
n \text { even, } \quad p \geqq 0 \text {, }
$$


(ii)

$$
\begin{array}{ll}
n \text { odd, } & p \geqq 0, \\
n \text { even, } & p \leqq 0, \\
n \text { odd, } & p \leqq 0 .
\end{array}
$$

Eq. (E) satisfying condition (i), for example, is denoted by $\left(\mathrm{E}_{\mathrm{i}}\right)$; $\left(\mathrm{E}_{\mathrm{ii}}\right)$, $\left(\mathrm{E}_{\mathrm{iii}}\right)$, and $\left(\mathrm{E}_{\mathrm{iv}}\right)$ are similarly defined.

We state important inequalities which will be used in defining the class $A_{p}$ and also in some proofs.

LEMMA 1. Let $y$ be a nonoscillatory solution of (E) such that $y \geqq 0$ on $[b, \infty)$ for some $b \geqq a$, and let $p \not \equiv 0$ on $\left[b_{1}, \infty\right)$ for every $b_{1} \geqq a$. Define $[C]$ to be the greatest integer less than or equal to $C$.

If $y$ is a solution of $\left(\mathrm{E}_{\mathrm{i}}\right)$ or $\left(\mathrm{E}_{\mathrm{iv}}\right)$, there exists an integer $j$, $0 \leqq j \leqq[(n-1) / 2]$, such that

$$
y^{(i)}>0, \quad i=0,1, \cdots, 2 j,
$$

on $\left[b_{2}, \infty\right)$ for some $b_{2} \geqq b$, and

$$
(-1)^{i+1} y^{(i)}>0, \quad i=2 j+1, \cdots, n-1,
$$

on $[b, \infty)$.

If $y$ is a solution of $\left(\mathrm{E}_{\mathrm{ii}}\right)$ or $\left(\mathrm{E}_{\mathrm{ii}}\right)$, there exists an integer $j$, $0 \leqq j \leqq[n / 2]$, such that

$$
y^{(i)}>0, \quad i=0,1, \cdots, 2 j-1,
$$

on $\left[b_{2}, \infty\right)$ for some $b_{2} \geqq b$, and

$$
(-1)^{i} y^{(i)}>0, \quad i=2 j, \cdots, n-1,
$$

on $[b, \infty)$.

Various versions of Lemma 1 appear in the literature [2, 5, 6, 12]. However, the important features of the present version are that the inequalities in Lemma 1 are strict and that the inequalities $\left(2^{\prime}\right)$ and $\left(3^{\prime}\right)$ hold on $[b, \infty)$-rather than on $\left[b_{2}, \infty\right)$ for some $b_{2} \geqq b$ if $y \geqq 0$ on $[b, \infty)$. Following Kiguradze [4], we shall say that a nonoscillatory solution $y$ of $\left(\mathrm{E}_{\mathrm{i}}\right)$ or $\left(\mathrm{E}_{\mathrm{iv}}\right)$ belongs to class $A_{j}$ if $y$ or $-y$ satisfies the inequalities $(2)$ and $\left(2^{\prime}\right)$ for $0 \leqq j \leqq[(n-1) / 2]$. Similarly, a nonoscillatory solution $y$ of $\left(\mathrm{E}_{\mathrm{ii}}\right)$ or $\left(\mathrm{E}_{\mathrm{iii}}\right)$ is said to belong to class $A_{j}$ if $y$ or $-y$ satisfies the inequalities (3) and $\left(3^{\prime}\right)$ for $0 \leqq$ $j \leqq[n / 2]$. In view of the above definition, we may restate Lemma 1 as follows: The family $\left\{A_{0}, A_{1}, \cdots, A_{[(n-1) / 2]}\right\}$ forms a partition of the nonoscillatory solutions of $\left(\mathrm{E}_{\mathrm{i}}\right)$ and $\left(\mathrm{E}_{\mathrm{iv}}\right)$, and the family $\left\{A_{0}, A_{1}, \cdots, A_{[n / 2]}\right\}$ forms a partition of the nonoscillatory solutions of 
$\left(\mathrm{E}_{\mathrm{ii}}\right)$ and $\left(\mathrm{E}_{\mathrm{iii}}\right)$.

LEMMA 2. If the class $A_{k}$ contains three solutions $v_{1}, v_{2}$, and $v_{3}$ of which every nontrivial linear combination again belongs to $A_{k}$, where $0 \leqq k \leqq[(n-2) / 2]$ for $\left(\mathrm{E}_{\mathrm{i}}\right)$ and $\left(\mathrm{E}_{\mathrm{iv}}\right)$ and $1 \leqq k \leqq[(n-1) / 2]$ for $\left(\mathrm{E}_{\mathrm{ii}}\right)$ and $\left(\mathrm{E}_{\mathrm{ii \textrm {i }}}\right)$, then $A_{k}$ contains three solutions $y_{1}, y_{2}$, and $y_{3}$, each a linear combination of $v_{1}, v_{2}$, and $v_{3}$, such that

$$
\lim _{x \rightarrow \infty} \frac{y_{j}(x)}{y_{i}(x)}=\infty, \quad 1 \leqq i<j \leqq 3
$$

Proof. Without loss of generality, we may assume that $v_{3}>$ $v_{2}>v_{1}>0$ on $[c, \infty)$ for some $c \geqq a$. The quotient $v_{j} / v_{i}, 1 \leqq i<j \leqq 3$, cannot assume a fixed value $\gamma$ an infinite number of times on $[c, \infty)$, for otherwise $v_{j}-\gamma v_{i}$ would be an oscillatory solution contrary to the hypothesis. Therefore,

$$
\limsup _{x \rightarrow \infty} \frac{v_{j}(x)}{v_{i}(x)}=\liminf _{x \rightarrow \infty} \frac{v_{j}(x)}{v_{i}(x)}=\lim _{x \rightarrow \infty} \frac{v_{j}(x)}{v_{i}(x)}=K_{i j},
$$

$1 \leqq K_{i j} \leqq \infty, 1 \leqq i<j \leqq 3$. At first there appear to be eight different possibilities we must consider, depending on $K_{i j}=\infty$ or $K_{i j}<\infty, 1 \leqq i<j \leqq 3$. But note that if two of the constants $K_{i j}$, $1 \leqq i<j \leqq 3$, are finite, the third also must be finite. Furthermore, it is impossible to have $K_{12}=K_{23}=\infty$ and $K_{13}<\infty$. Hence we need only to consider the following four cases.

(a) $K_{i j}=\infty, 1 \leqq i<j \leqq 3$. Put $y_{i}=v_{i}, i=1,2,3$.

(b) $K_{12}<\infty, K_{13}=K_{23}=\infty$. In this case

$$
\lim _{x \rightarrow \infty} \frac{v_{2}(x)-K_{12} v_{1}(x)}{v_{1}(x)}=0 \text {, i.e., } \lim _{x \rightarrow \infty}\left|\frac{v_{1}(x)}{v_{2}(x)-K_{12} v_{1}(x)}\right|=\infty \text {. }
$$

Put $y_{1}=v_{2}-K_{12} v_{1}, y_{2}=v_{1}$, and $y_{3}=v_{3}$.

(c) $K_{12}=K_{13}=\infty, K_{23}<\infty$. Here we have

$$
\lim _{x \rightarrow \infty} \frac{v_{3}(x)-K_{23} v_{2}(x)}{v_{2}(x)}=0 \text {. }
$$

Suppose that

$$
\lim _{x \rightarrow \infty} \frac{v_{3}(x)-K_{23} v_{2}(x)}{v_{1}(x)}=K
$$

If $|K|=\infty$, put $y_{1}=v_{1}, y_{2}=v_{3}-K_{23} v_{2}$, and $y_{3}=v_{2}$. On the other hand, if $|K|<\infty$, then

$$
\lim _{x \rightarrow \infty} \frac{v_{3}(x)-K_{23} v_{2}(x)-K v_{1}(x)}{v_{1}(x)}=0
$$


and we put $y_{1}=v_{3}-K_{23} v_{2}-K v_{1}, y_{2}=v_{1}$, and $y_{3}=v_{2}$.

(d) $K_{i j}<\infty, 1 \leqq i<j \leqq 3$. For this case

$$
\lim _{x \rightarrow \infty} \frac{v_{2}(x)-K_{12} v_{1}(x)}{v_{1}(x)}=\lim _{x \rightarrow \infty} \frac{v_{3}(x)-K_{13} v_{1}(x)}{v_{1}(x)}=0 .
$$

Suppose that

$$
\lim _{x \rightarrow \infty} \frac{v_{2}(x)-K_{12} v_{1}(x)}{v_{3}(x)-K_{13} v_{1}(x)}=K
$$

If $|K|=\infty$, let $y_{1}=v_{3}-K_{13} v_{1}, y_{2}=v_{2}-K_{12} v_{1}$, and $y_{3}=v_{1}$. If $|K|<\infty$, then

$$
\lim _{x \rightarrow \infty} \frac{v_{2}(x)-K_{12} v_{1}(x)-K\left(v_{3}(x)-K_{13} v_{1}(x)\right)}{v_{3}(x)-K_{13} v_{1}(x)}=0
$$

and we put $y_{1}=v_{2}-\left(K_{12}-K K_{13}\right) v_{1}-K v_{3}, y_{2}=v_{3}-K_{13} v_{1}$, and $y_{3}=v_{1}$. The solutions $y_{i}, i=1,2,3$, defined in (a)-(d) belong to $A_{k}$ and satisfies

$$
\lim _{x \rightarrow \infty}\left|\frac{y_{j}(x)}{y_{i}(x)}\right|=\infty, \quad 1 \leqq i<j \leqq 3 .
$$

Since we may take $-y_{i}$ if $y_{i}$ is eventually negative as $x \rightarrow \infty$, the proof is complete.

Lemma 3. Suppose that Eq. (E) has there nonoscillatory solutions $y_{1}, y_{2}$, and $y_{3}$ such that

$$
\lim _{x \rightarrow \infty} \frac{y_{j}(x)}{y_{i}(x)}=\infty, \quad 1 \leqq i<j \leqq 3,
$$

and $y_{3}>y_{2}>y_{1}>0$ on $[\xi, \infty)$. If $\eta$ is an arbitrary point on $[\xi, \infty)$, there exists a solution $v \equiv \sum_{k=1}^{3} \alpha_{k} y_{k}$ such that $v \geqq 0$ on $[\xi, \infty)$ and $v(\zeta)=v^{\prime}(\zeta)=0$ for some point $\zeta$ on $[\eta, \infty)$.

Proof. Choose a constant $K>0$ such that $u \equiv y_{2}-K y_{1}<0$ on $[\xi, \eta]$. Since $u<0$ on $[\xi, \eta]$ and eventually $u(x)>0$ as $x \rightarrow \infty, u$ vanishes at some point of $(\eta, \infty)$. Let $\sigma$ be the first zero of $u$ on $(\eta, \infty)$. Define $K_{1}=\sup G$, where $G$ is the set of real numbers $\beta \geqq 1$ such that $y_{3}-\beta u \geqq 0$ on $[\sigma, \infty)$. Evidently, $G$ is bounded above and it is nonempty because $y_{3}-u>0$, i.e., $1 \in G$. Let $\beta \in G$ and $\tau \in[\sigma, \infty)$. If $u(\tau) \leqq 0$, then $y_{3}(\tau)-K_{1} u(\tau) \geqq y_{3}(\tau)>0$. On the other hand, if $u(\tau)>0$, then $y_{3}(\tau) / u(\tau) \geqq \beta$ for all $\beta \in G$, and thus $y_{3}(\tau) / u(\tau) \geqq K_{1}$. Since $\tau$ is arbitrary, the solution $v \equiv y_{3}-K_{1} u \geqq 0$ on $[\sigma, \infty)$. Therefore, if $v(\zeta)=0$ for some $\zeta \in(\sigma, \infty)$, then $v^{\prime}(\zeta)=0$. Hence, the proof is complete if we can show that $v(\zeta)=0$ for some $\zeta \in(\sigma, \infty)$. Assume 
to the contrary that $v>0$ on $(\sigma, \infty)$. Let $\varepsilon_{1}>0$ be given. There exists $\rho>\sigma$ such that $u>0$ on $[\rho, \infty)$ and $v(x) / u(x)>\varepsilon_{1}, x \in[\rho, \infty)$, since

$$
\lim _{x \rightarrow \infty} \frac{v(x)}{u(x)}=\infty
$$

by (4). Choose an $\varepsilon_{2}>0$ so that $v(x)>\varepsilon_{2} u(x), x \in[\sigma, \rho]$. Put $\varepsilon=$ $\min \left(\varepsilon_{1}, \varepsilon_{2}\right)$. Then $v-\varepsilon u>0$ on $[\sigma, \infty)$, i.e., $y_{3}-\left(K_{1}+\varepsilon\right) u>0$ on $[\sigma, \infty)$, contradicting the choice of $K_{1}$. Thus, $v(\zeta)=0$ for some $\zeta \in(\sigma, \infty)$. Finally, it is evident that $v>0$ on $[\xi, \sigma]$ and $v \geqq 0$ on $[\xi, \infty)$.

We are ready to consider the problem of determining the number of solutions belonging to class $A_{j}$. Let $q\left(A_{j}\right)$ be the maximum number of linearly independent solutions belonging to $A_{j}$ with the property that every nontrivial linear combination of them again belongs to class $A_{j}$.

TheOREM. Assume that Eq. (E) is nonoscillatory on $[a, \infty)$ and that $p \not \equiv 0$ on $\left[a_{1}, \infty\right)$ for every $a_{1} \geqq a$. Then

$$
\begin{aligned}
& q\left(A_{j}\right)=2, j=0,1, \cdots,(n-2) / 2, \text { for }\left(\mathrm{E}_{\mathrm{i}}\right) ; \\
& q\left(A_{0}\right)=1, q\left(A_{j}\right)=2, j=1,2, \cdots,(n-1) / 2, \text { for }\left(\mathrm{E}_{\mathrm{ii}}\right) ; \\
& q\left(A_{0}\right)=1, q\left(A_{j}\right)=2, j=1,2, \cdots,(n-2) / 2, q\left(A_{n / 2}\right)=1, \text { for }\left(\mathrm{E}_{\mathrm{ii1}}\right) ; \\
& q\left(A_{j}\right)=2, j=0,1, \cdots,(n-3) / 2, q\left(A_{(n-1) / 2}\right)=1 \text { for }\left(\mathrm{E}_{\mathrm{iv}}\right) .
\end{aligned}
$$

Proof. We shall prove the theorem for $\left(\mathrm{E}_{\mathrm{iii}}\right): q\left(A_{0}\right)=1, q\left(A_{j}\right)=2$, $j=1,2, \cdots,(n-2) / 2$, and $q\left(A_{n / 2}\right)=1$. Suppose that the class $A_{j}$ contains a set $B_{j}$ of $q\left(A_{j}\right)$ solutions of which every nontrivial linear combination again belongs to $A_{j}, j=0,1, \cdots, n / 2$. Using Lemmas 1 and 2 , we can easily deduce that the set $B=\bigcup_{j=0}^{n / 2} B_{j}$ containing $\sum_{j=0}^{n / 2} q\left(A_{j}\right)$ solutions is a fundamental system for $\left(\mathrm{E}_{\mathrm{iij}}\right)$. Thus, $\sum_{j=0}^{n / 2} q\left(A_{j}\right)=n$. For this reason, it suffices to prove that

$$
q\left(A_{0}\right) \leqq 1, \quad q\left(A_{j}\right) \leqq 2, \quad j=1,2, \cdots,(n-2) / 2, q\left(A_{n / 2}\right) \leqq 1 .
$$

If $q\left(A_{0}\right)>1$, then there exist two solutions $y_{1}$ and $y_{2}$ belonging to $A_{0}$ and a constant $K$ such that $w \equiv y_{1}-K y_{2} \in A_{0}, w(c)=0$, and $w \geqq 0$ on $[c, \infty)$ for some $c \geqq a$. But this contradicts Lemma 1 (see also Kiguradze [5, Lemma 7]) and proves that $q\left(A_{0}\right) \leqq 1$. Suppose that $q\left(A_{k}\right)>2$ for some $k, 1 \leqq k \leqq(n-2) / 2$. Then the class $A_{k}$ contains at least three solutions $y_{1}, y_{2}$, and $y_{3}$, of which every nontrivial linear combination again belongs to $A_{k}$. By Lemma 2, we may assume that

$$
\lim _{x \rightarrow \infty} \frac{y_{j}(x)}{y_{i}(x)}=\infty, \quad 1 \leqq i<j \leqq 3,
$$


and $y_{3}>y_{2}>y_{1}>0$ on $[\xi, \infty)$ for some $\xi \geqq a$. Let $\left\{\eta_{i}\right\}$ be an increasing sequence of numbers such that $\eta_{i} \geqq \xi$ and $\eta_{i} \rightarrow \infty$ as $i \rightarrow \infty$. By Lemma 3 there exists for each $i$, a solution

$$
v_{i} \equiv \alpha_{i} y_{1}+\beta_{i} y_{2}+\gamma_{i} y_{3}, \quad \alpha_{i}^{2}+\beta_{i}^{2}+\gamma_{i}^{2}=1,
$$

such that $v_{i} \geqq 0$ on $[\xi, \infty)$ and $v_{i}\left(\zeta_{i}\right)=v_{i}^{\prime}\left(\zeta_{i}\right)=0$ for some $\zeta_{i} \in\left(\eta_{i}, \infty\right)$. Obviously, there are convergent subsequences $\left\{\alpha_{i_{k}}\right\},\left\{\beta_{i_{k}}\right\}$, and $\left\{\gamma_{i_{k}}\right\}$, which will be again denoted by $\left\{\alpha_{i}\right\},\left\{\beta_{i}\right\}$, and $\left\{\gamma_{i}\right\}$, respectively, for notational simplicity. Put

$$
\lim _{i \rightarrow \infty} \alpha_{i}=\alpha, \quad \lim _{i \rightarrow \infty} \beta_{i}=\beta, \quad \lim _{i \rightarrow \infty} \gamma_{i}=\gamma .
$$

Then $w(x) \equiv \alpha y_{1}(x)+\beta y_{2}(x)+\gamma y_{3}(x)$ is a nonoscillatory solution belonging to the class $A_{k}$. Since $w \geqq 0$ on $[\xi, \infty)$, we have

$$
w>0, w^{\prime}>0, \cdots, w^{(2 k-1)}>0,
$$

on $\left[b_{2}, \infty\right)$ for some $b_{2} \geqq \xi$, and

$$
w^{(2 k)}>0, \quad w^{(2 k+1)}<0, \quad w^{(2 k+2)}>0, \cdots, w^{(n-1)}<0,
$$

on $[\xi, \infty)$ by Lemma 1 . We now use a line of reasoning due to Kondrat'ev [7]. Since $\lim _{i \rightarrow \infty} v_{i}^{(j)}=w^{(j)}, j=0,1, \cdots, n$, uniformly on any finite subinterval of $[a, \infty)$, there exists a number $N$ such that

$$
v_{i}^{(j)}\left(b_{2}\right)>\frac{w^{(j)}\left(b_{2}\right)}{2}>0, \quad j=0,1, \cdots, 2 k-1,
$$

if $i>N$. We may assume that $\eta_{i}>b_{2}$ for $i>N$. Since $v_{i} \in A_{k}$ and $v_{i} \geqq 0$ on $[\xi, \infty)$ for all $i, v_{i}^{(2 k)}>0$ on $[\xi, \infty)$ by Lemma 1 . Thus,

$$
v_{i}^{(2 k-1)}\left(b_{2}\right) \leqq v_{i}^{(2 k-1)}(\tau), \quad \tau \in\left[b_{2}, \infty\right) .
$$

Substituting (9) in (8) with $j=2 k-1$, we obtain

$$
v_{i}^{(2 k-1)}(\tau)>\frac{w^{(2 k-1)}\left(b_{2}\right)}{2}, \quad \tau \in\left[b_{2}, \infty\right) .
$$

Integrating the above inequality from $b_{2}$ to $x \in\left[b_{2}, \infty\right)$ and substituting in the resulting expression the inequality (8) with $j=2 k-2$, we get

$$
v_{i}^{(2 k-2)}(x)>\frac{w^{(2 k-1)}\left(b_{2}\right)}{2}\left(x-b_{2}\right)+\frac{w^{(2 k-2)}\left(b_{2}\right)}{2} .
$$

Repeating a similar procedure $2 k-2$ times, we arrive at the inequality 


$$
\begin{aligned}
v_{i}(x)> & \frac{w^{(2 k-1)}\left(b_{2}\right)}{2(2 k-1) !}\left(x-b_{2}\right)^{2 k-1}+\frac{w^{(2 k-2)}\left(b_{2}\right)}{2(2 k-2) !}\left(x-b_{2}\right)^{2 k-2} \\
& +\cdots+\frac{w\left(b_{2}\right)}{2}, \quad x \in\left[b_{2}, \infty\right) .
\end{aligned}
$$

This inequality, however, cannot hold throughout the interval $\left[b_{2}, \infty\right)$. Indeed, for $x=\zeta_{i}>\eta_{i}>b_{2}(i>N)$, the left-hand side $v_{i}\left(\zeta_{i}\right)=0$, while the right-hand side is positive by (6). This contradiction proves that $q\left(A_{j}\right) \leqq 2, j=1,2, \cdots,(n-2) / 2$. The proof that $q\left(A_{n / 2}\right) \leqq 1$ is more or less similar to the preceding case. Suppose that $A_{n / 2}$ contains two solutions $y_{1}$ and $y_{2}$ of which every nontrivial linear combination belongs to $A_{n / 2}$. Assume that $y_{2}>y_{1}>0$ on $[\xi, \infty)$, and let $\left\{\eta_{i}\right\}$ be defined as before. Put

$$
v_{i} \equiv \alpha_{i} y_{1}+\beta_{i} y_{2}, \quad \alpha_{i}^{2}+\beta_{i}^{2}=1,
$$

such that $v_{i}\left(\eta_{i}\right)=0$. If

$$
\lim _{i \rightarrow \infty} \alpha_{i}=\alpha, \quad \lim _{i \rightarrow \infty} \beta_{i}=\beta
$$

(take subsequences, if necessary), define $w \equiv \alpha y_{1}+\beta y_{2}$. Then $w \in A_{n / 2}$ and we may assume that $w \geqq 0$ on $[b, \infty)$ for some $b$. Hence, by Lemma 1,

$$
w>0, w^{\prime}>0, \cdots, w^{(n-1)}>0,
$$

on $\left[b_{2}, \infty\right)$ for some $b_{2} \geqq b$, and the inequality (8) holds for $i>N_{1}$, for some $N_{1}$, and for $j=0,1, \cdots, n-1$. Assume that $\eta_{i}>b_{2}$ for $i>N_{1}$. For each $i>N_{1}$, there exists $c_{i} \in\left(b_{2}, \eta_{i}\right]$ such that $v_{i}\left(c_{i}\right)=0$ and $v_{i}>0$ on $\left[b_{2}, c_{i}\right)$, since $v_{i}\left(\eta_{i}\right)=0$. On the interval $\left[b_{2}, c_{i}\right]$, we have $v_{i}^{(n)}(x)=-p(x) v_{i}(x) \geqq 0$. Therefore, $v_{i}^{(n-1)}\left(b_{2}\right) \leqq v_{i}^{(n-1)}(\tau), \tau \in\left[b_{2}, c_{i}\right]$, and when this inequality is substituted in (8) with $j=n-1$, we get

$$
v_{i}^{(n-1)}(\tau)>\frac{w^{(n-1)}\left(b_{2}\right)}{2}, \quad \tau \in\left[b_{2}, c_{i}\right] .
$$

Following the procedure employed to get from (10) to (11), we alternately integrate (13) from $b_{2}$ to $x \in\left[b_{2}, c_{i}\right]$ and substitute in the resulting expression a suitable inequality from (8) (which holds for $j=0,1, \cdots, n-1$, in this case). When this process is repeated $n-1$ times, we arrive at

$$
\begin{aligned}
v_{i}(x)> & \frac{w^{(n-1)}\left(b_{2}\right)}{2(n-1) !}\left(x-b_{2}\right)^{n-1}+\frac{w^{(n-2)}\left(b_{2}\right)}{2(n-2) !}\left(x-b_{2}\right)^{n-2} \\
& +\cdots+\frac{w\left(b_{2}\right)}{2}, \quad x \in\left[b_{2}, c_{i}\right] .
\end{aligned}
$$

However, this inequality cannot hold at $x=c_{i}$ because $v_{i}\left(c_{i}\right)=0$ 
while the right-hand side is positive by virtue of (12). Consequently, $q\left(A_{n / 2}\right) \leqq 1$, and the proof is complete for $\left(\mathrm{E}_{\mathrm{iii}}\right)$. Proofs for $\left(\mathrm{E}_{\mathrm{i}}\right),\left(\mathrm{E}_{\mathrm{ii}}\right)$, and $\left(\mathrm{E}_{\mathrm{iv}}\right)$ are similar.

This theorem generalizes a main result of Etgen and Taylor [3].

\section{REFERENCES}

1. G. V. Anan'eva and V. I. Balaganski, Oscrllation of the solutions of certain differential equations of higher order, Uspehi Mat. Nauk, 14 (1959), 135-140.

2. G. A. Bogar, Oscillations of $n$th order differential equations with retarded argument, SIAM J. Math. Anal., 5 (1974), 473-481.

3. G. J. Etgen and W. E. Taylor, Jr., The essential uniqueness of bounded nonoscillatory solutions of certain even order differential equations, Pacific J. Math., 68 (1977), 339-346. 4. I. T. Kiguradze, Oscillation properties of solutions of certain ordinary differential equations, Dokl. Akad. Nauk, 144 (1962), 33-36.

5. - On the oscillatory character of solutions of the equation $d^{m} u / d t^{m}+$ $a(t)|u|^{n} \operatorname{sign} u=0$, Mat. Sb., 65 (107) (1964), 172-187.

6. W. J. Kim, Nonoscillatory solutions of a class of $n$ th-order linear differential equations,

J. Differential Equations, 27 (1978), 19-27.

7. V. A. Kondrat'ev, Oscillatory properties of solutions of the equation $y^{(n)}+p y=0$, Trudy Moskov. Mat. Obsc, 10 (1961), 419-436.

8. J. G.-Mikusinski, On Fite's oscillation theorem, Colloq. Math., 2 (1949), 34-39.

9. Z. Nehari, Disconjugacy criteria for linear differential equations, J. Differential Equations, 4 (1968), 604-611.

10. - Nonlinear techniques for linear oscillation problems, Trans. Amer. Math. Soc., 210 (1975), 387-406.

11. - Green's functions and disconjugacy, Arch. Rational Mech. Anal., 62 (1976), 53-76.

12. G. H. Ryder and D. V. V. Wend, Oscillation of solutions of certain differential equations of $n$th order, Proc. Amer. Math. Soc., 25 (1970), 463-469.

13. I. M. Sobol', On the asymptotic behavior of the solutions of linear differential equations, Dokl. Akad. Nauk SSSR, 61 (1948), 219-222.

14. W. F. Trench, A sufficient condition for eventual disconjugacy, Proc. Amer. Math. Soc., 52 (1975), 139-146.

15. D. Willett, Disconjugacy tests for singular linear differential equations, SIAM J. Math. Anal., 2 (1971), 536-545.

Received March 19, 1979.

State University of New York

Stony BRook, NY 11794 


\section{PACIFIC JOURNAL OF MATHEMATICS}

\section{EDITORS}

DONALD BABBITT (Managing Editor)

University of Galifornia

Los Angeles, California 90024

HUgo RossI

University of Utah

Salt Lake City, UT 84112

C. C. MOORE AND ANDREW OGG

University of California

Berkeley, CA 94720

\section{J. DUGUNDJI}

Department of Mathematics University of Southern California Los Angeles, California 90007

R. Finn and J. Milgram

Stanford University

Stanford, California 94305

\section{ASSOCIATE EDITORS}
R. ARENS
E. F. BECKENBACH
B. H. NeUManN
F. WOLF
K. YosHIDA

\section{SUPPORTING INSTITUTIONS}

UNIVERSITY OF ARIZONA

UNIVERSITY OF BRITISH COLUMBIA

CALIFORNIA INSTITUTE OF TECHNOLOGY

UNIVERSITY OF CALIFORNIA

MONTANA STATE UNIVERSITY

UNIVERSITY OF NEVADA, RENO

NEW MEXICO STATE UNIVERSITY

OREGON STATE UNIVERSITY
UNIVERSITY OF OREGON UNIVERSITY OF SOUTHERN CALIFONIA STANFORD UNIVERSITY UNIVERSITY OF HAWAII UNIVERSITY OF TOKYO UNIVERSITY OF UTAH WASHINGTON STATE UNIVERSITY UNIVERSITY OF WASHINGTON 


\section{Pacific Journal of Mathematics}

\section{Vol. 93, No. $1 \quad$ March, 1981}

Richard Arens, Reducing the order of a Lagrangian $\ldots \ldots \ldots \ldots \ldots \ldots \ldots$

Richard Arens, Manifestly dynamic forms in the Cartan-Hamilton treatment

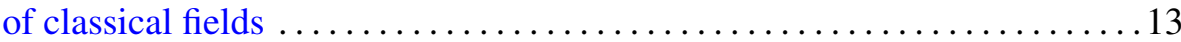

Jimmy T. Arnold, Power series rings over discrete valuation rings $\ldots \ldots \ldots 31$

Charles A. Asmuth and Joe Repka, Supercuspidal components of the

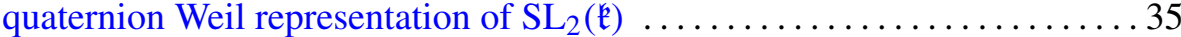

Luis A. Caffarelli and Avner Friedman, Sequential testing of several simple hypotheses for a diffusion process and the corresponding free boundary problem ................................. 49

William B. Jacob, Fans, real valuations, and hereditarily-Pythagorean fields .............................................. 95

W. J. Kim, Asymptotic properties of nonoscillatory solutions of higher order

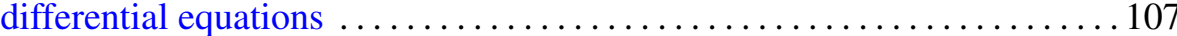

Wayne Steven Lewis, Embeddings of the pseudo-arc in $E^{2} \ldots \ldots \ldots \ldots \ldots 115$

Daniel Alan Marcus, Closed factors of normal Z-semimodules . ......... 121

Mitsuru Nakai and Leo Sario, Harmonic functionals on open Riemann surfaces ............................................. 147

John Currie Quigg, Jr., On the irreducibility of an induced

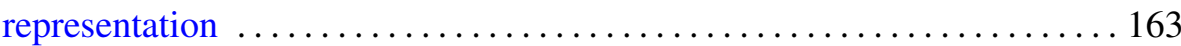

John Henry Reinoehl, Lie algebras and Hopf algebras 181

Joe Repka, Base change for tempered irreducible representations of $\mathrm{GL}(n, \mathbf{R})$

Peter John Rowley, Solubility of finite groups admitting a fixed-point-free automorphism of order $r s t$. I . . . . . . . . . . . . . . . . . . 201

Alan C. Woods, The asymmetric product of three homogeneous linear forms 